\title{
Comparative diagnostic value of ultrasound, ultrasound-guided fine needle aspiration and sestamibi scintigraphy for the correct preoperative localisation of parathyroid adenomas
}

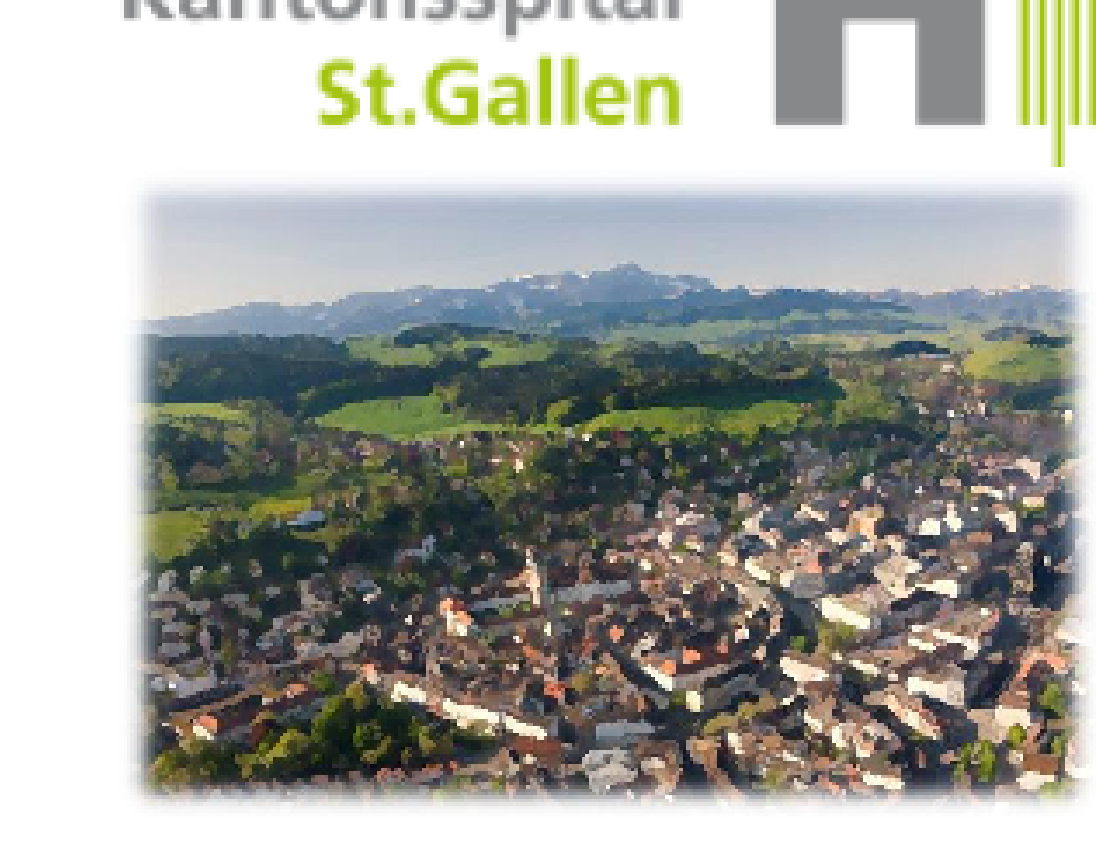

\author{
S. Bilz ${ }^{1}$, N. Rogowski-Lehmann ${ }^{1}$, I. Krull ${ }^{1}$, R. Oettli², M. Brändle ${ }^{1}$, W. Kolb ${ }^{3}$, T. Clerici $^{3}$ \\ ${ }^{1}$ Division of Endocrinology, Department of Internal Medicine, ${ }^{2}$ Department of Radiology and Nuclear Medicine and ${ }^{3}$ Department of Surgery, \\ Kantonssspital St. Gallen, St. Gallen, Switzerland
}

\section{INTRODUCTION AND OBJECTIVES}

Open minimally invasive parathyroidectomy (OMIP) has become the standard surgical therapy for patients with primary hyperparathyroidism ( $\mathrm{HHPT}$ ) and requires exact preoperative localisation procedures.

This study prospectively assessed the sensitivity and positive predictive value (PPV) of ultrasound (US), ultrasound-guided fine needle aspiration with PTH measurement in the needle washout (US-FNA) and sestamibi scintigraphy (SS) for the localisation of parathyroid adenomas in patients with $\mathrm{PHPT}$

\section{METHODS}

51 consecutive patients with pHPT were included. Patients with clinical criteria indicative of multiglandular disease were not eligible. US and US-FNA were performed by 2 endocrinologists using highresolution ultrasound and a free hand technique. A lesion was considered suspect for a parathyroid adenoma if $\geq 4$ of the following criteria were present: hypoechoic pattern, presence a sharp margin, round, oval or triangular shape, compatible/typical location and presence of a vascular pedicle using color-flow imaging.

For US-FNA, a 24 or $25 \mathrm{G}$ needle attached to a $10 \mathrm{cc}$ syringe in a pistol was placed in the suspected lesion with ultrasound guidance and slight suction was applied while the needle was gently moved forth and back 4-5 times in the lesion under continued ultrasound control. The needle and syringe were then rinsed with $3 \mathrm{cc}$ of saline in which the PTH concentration was measured. A US-FNA was considered positive, if the PTH concentration in the aspirate was $>$ 2 fold increased when compared to a simultaneously measured plasma sample.

Double isotope scanning with $99 \mathrm{mTc}$ pertechnetate and $99 \mathrm{mTc}$ sestamibi was used during the scintigraphic studies. A localisation procedure was considered correct if surgical removal of a parathyroid gland at this localisation resulted in biochemical cure of the pHPT at 6 weeks and/or 6 months postoperatively. All surgeries were performed by 2 experienced endocrine surgeons using intraoperative PTH monitoring.

Data are given as mean standard error if not otherwise indicated. McNemar tests were used to test for significant differences between the different localisation procedures. Since all patients had a final diagnosis of PHPT secondary to uniglandular disease there were no correct-negative test results for SC and US.

\section{FIGURE 1:}
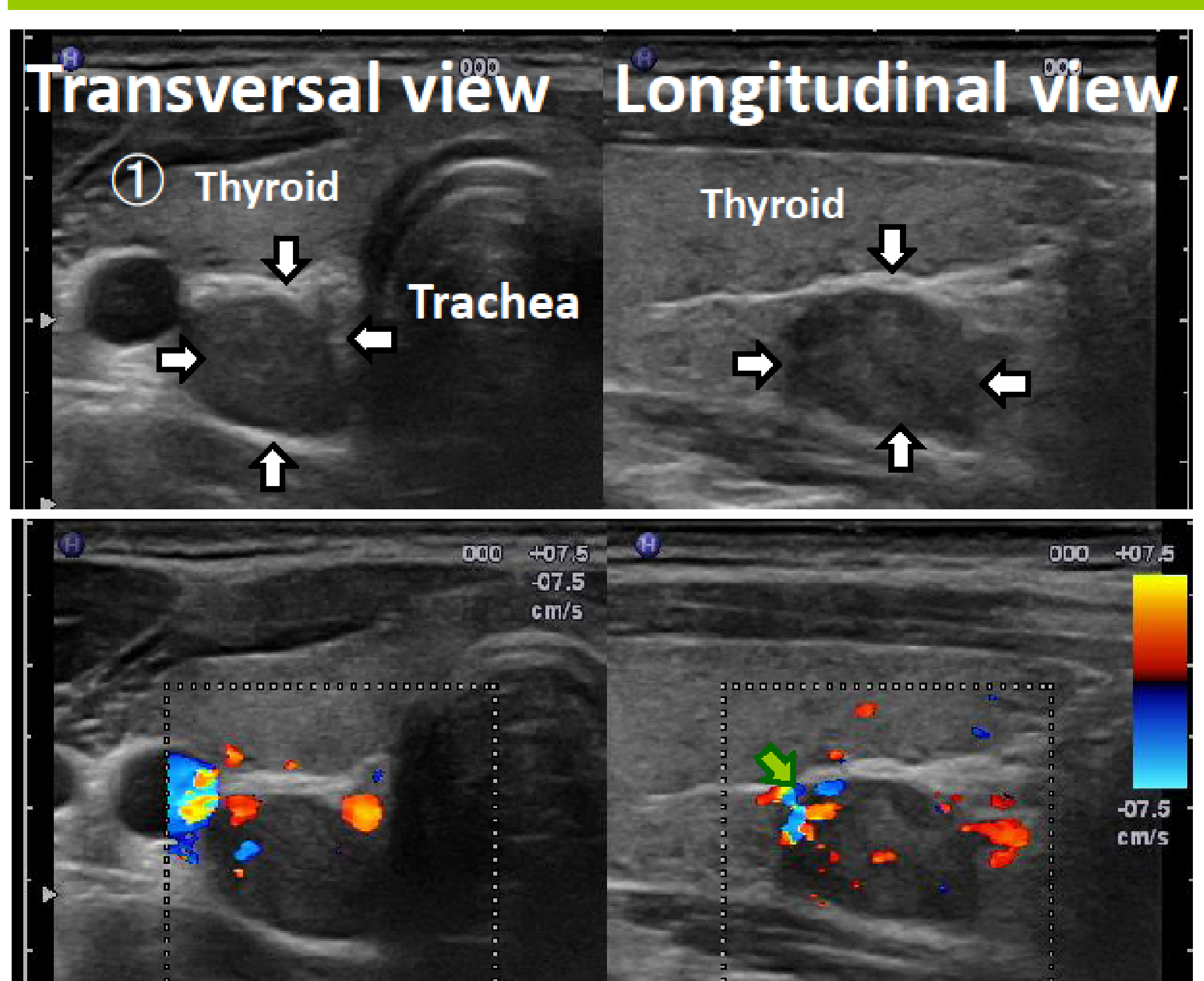

US image of a typical parathyroid adenoma (white arrows) with hypoechoic pattern, oval shape, sharp margins and a vascular pedicle indicated by color flow sonography (green arrow), located dorsal of the lower portion of the right thyroid lobe. (1) Common carotid artery.

\section{TABLE 1: BASELINE CHARACTERISTICS}

\section{Gender}

Age (years)

BMI $\left(\mathrm{kg} / \mathrm{m}^{2}\right)$

$\mathrm{SBP}(\mathrm{mmHg})$

DBP ( $\mathrm{mmHg}$ )

HR (bpm)

Osteopenia/-porosis (\%)

Urolithiasis (\%)

\section{$41 \mathrm{~F}, 10 \mathrm{M}$ Calcium $(\mathrm{mmol} / \mathrm{l})$}

$\mathbf{5 1} \pm \mathbf{2 . 0}$ Corr. Calcium ( $\mathrm{mmol} / \mathrm{l})$

$26 \pm 0.7$ lonized Calcium (mmol/l)

$142 \pm 2.2$ Phosphate $(\mathrm{mmol} / \mathrm{l})$

$82 \pm 1.7 \quad \mathrm{PTH}(\mathrm{ng} / \mathrm{l})$

$74 \pm 1.6 \quad 25-\mathrm{OH}$ Vitamin D3 (nmo/l)

25/51 Creatinin ( $\mathrm{mmol} / \mathrm{l})$

26 eGFR $(\mathrm{ml} / \mathrm{min})$
$2.71 \pm 0.03$

$2.73 \pm 0.03$

$1.45 \pm 0.02$

$0.85 \pm 0.02$

$113 \pm 6$

$68 \pm 3.6$

$73 \pm 2.4$

$93 \pm 3.5$
RESULTS

The baseline characteristics of the study population are given in table 1.

Biochemical cure indicated by normal plasma calcium concentrations 6 months postoperatively was achieved after the first surgery in 49 patients and in 2 after a second surgery. OMIP was successful in 39 subjects, a unilateral exploration was performed in 4 subjects (all b/o concomitant thyroid pathology) and 7 subjects were cured after a bilateral neck exploration. A thoracotomy was required in 2 patients with ectopic adenomas.

The overall accuracy of US was significantly higher when compared to SS $(p<0.02)$ and US-FNA $(p<0.03)$, although the latter tests had very high positive predictive value. SS failed to detect a significant portion of small adenomas and only $39 \%$ of small (volume by US < $0.25 \mathrm{ml}$ ) and US-negative adenomas were correctly located by SS. SS and US were discordant in $33 \%$ of the patients, mostly due to false negative SS (65\%). SS correctly located an adenoma in 2 of 4 US negative cases and in none of the 4 cases when US was false positive. US-FNA correctly classified $53 \%$ of the SS negative/US positive lesions. However, similar to SS, US-FNA showed a high falsenegative rate in small lesions and only $47 \%$ of the adenomas $<0.25$ $\mathrm{ml}$ by ultrasound were correctly classified.

Intraoperatively, fibrosis or scarring and hematomas were observed in 16 and $12 \%$ of the adenomas that had undergone US-FNA, respectively. In 3 cases the postprocedural changes were felt to significantly interfere with surgery.

\begin{tabular}{|c|c|c|c|}
\hline & Sensitivity & PPV & Accuracy \\
\hline Scintigraphy & 0.65 & 0.94 & 0.63 \\
\hline Ultrasound & 0.91 & 0.89 & 0.82 \\
\hline US-FNA & 0.68 & 0.96 & 0.69 \\
\hline
\end{tabular}

\section{CONCLUSIONS}

When compared to SS, US shows a superior sensitivity to detect parathyroid adenomas due to its ability to detect small lesions and should be used as a first line test. US-FNA may be considered in cases with discordant imaging results. Due to its limited sensitivity, SS may be reserved for US-negative cases. However, US by itself will allow OMIP in $90 \%$ of cases and directly proceeding to surgery or the use of novel imaging approaches such as $18 \mathrm{~F}$-choline-PET-CT may be alternative strategies in US negative cases. 